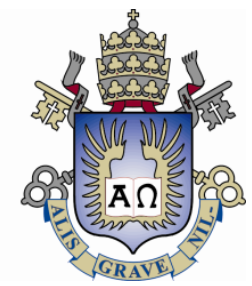

Claudio Veloso Barreto

\title{
Simulação Numérica do Escoamento em Estações de Entrega de Gás Natural
}

Dissertação apresentada ao Programa de Pósgraduação como requisito parcial para obtenção do título de Mestre em Engenharia Mecânica.

Orientador: Prof. Angela Ourivio Nieckele Co-Orientador: Luis Fernando Gonçalves Pires

Rio de Janeiro Fevereiro de 2011 
Claudio Veloso Barreto

\title{
Simulação Numérica do Escoamento em Estações de Entrega de Gás Natural
}

\begin{abstract}
Dissertação apresentada como requisito parcial para obtenção do título de Mestre pelo Programa de PósGraduação em Engenharia Mecânica da PUC-Rio. Aprovada pela Comissão Examinadora abaixo assinada.
\end{abstract}

Prof. Angela Ourivio Nieckele Orientadora Pontifícia Universidade Católica do Rio de Janeiro

Dr. Luis Fernando Gonçalves Pires Co-Orientador Instituto de Pesquisa e Desenvolvimento - Ctex

\section{Dr. João Paulo de Barros Leite TRANSPETRO S.A}

Prof. José Alberto dos Reis Parise Pontifícia Universidade Católica do Rio de Janeiro

Prof. José Eugênio Leal Coordenador Setorial do Centro

Técnico Científico - PUC-Rio 
Todos os direitos reservados. É proibida a reprodução total ou parcial do trabalho sem autorização da universidade, do autor e do orientador.

Claudio Veloso Barreto

Graduou-se em Engenharia Industrial Mecânica no CEFET-RJ (Centro Federal de Educação Tecnológica Celso Suckow da Fonseca) em 2003. É coordenador de pesquisa do laboratório SIMDUT (Núcleo de Simulação Termohidráulica de Dutos) vinculado ao Departamento de Engenharia Mecânica da PUC-Rio. Instrutor no curso de simulação de escoamento em dutos oferecido anualmente pelo CCE/PUC-Rio.

Ficha Catalográfica

Barreto, Claudio Veloso

Simulação numérica do escoamento em estações de entrega de gás natural / Claudio Veloso Barreto ; orientadores: Angela Ourivio Nieckele, Luis Fernando Gonçalves Pires. 2011.

139 f. : il. (color.) ; $30 \mathrm{~cm}$

Dissertação (mestrado)-Pontifícia

Universidade Católica do Rio de Janeiro, Departamento de Engenharia Mecânica, 2011.

Inclui bibliografia

1. Engenharia mecânica - Teses. 2. Estações de entrega de gás natural.3. Simulação de processos dinâmicos. 4. Aquecedores de gás indiretos de banho de água. I. Nieckele, Angela Ourivio. II. Pires, Luis Fernando Gonçalves. III. Pontifícia Universidade Católica do Rio de Janeiro. Departamento de Engenharia Mecânica. IV. Título.

CDD: 621 


\section{Agradecimentos}

A minha esposa Daniele, pelo amor, carinho e dedicação imensuráveis.

A minha filha Sarah, fonte de alegria e conforto nas horas em que precisei recarregar as baterias.

Aos meus pais Claudio e Maria Inez, que me ensinaram o valor da família e apoio para minha formação acadêmica.

Aos professores orientadores Luis Fernando G. Pires e Angela O. Nieckele pela orientação e pela oportunidade.

A todos os professores que compuseram o quadro de aulas oferecidas.

Ao Departamento de Engenharia Mecânica da PUC-Rio.

A Transportadora Brasil-Bolívia (TBG) e a Petrobrás S.A por viabilizarem a pesquisa e desenvolvimento do simulador e criação do tema desta dissertação.

E a todos que, de alguma forma, me ajudaram a concluir mais este objetivo. 


\section{Resumo}

Barreto, Claudio Veloso; Nieckele, Angela O. Nieckele; Pires, Luis Fernando G. Simulação Numérica do Escoamento em Estações de Entrega de Gás Natural, 2011. 139p. Dissertação de Mestrado Departamento de Engenharia Mecânica, Pontifícia Universidade Católica do Rio de Janeiro.

Análise numérica do processo dinâmico do escoamento de gás natural em estações de entrega, considerando o comportamento transiente dos principais equipamentos existentes, tais como válvula de três vias, válvulas redutoras de pressão e aquecedores de gás. Desenvolvimento da lógica de controle dinâmica (controle PID - proporcional-integral-derivativo) de todos os equipamentos relevantes. Para determinação das propriedades termodinâmicas do gás natural empregou-se as equações de estado Peng-Robinson e Soave-Redlich-Kwong. O estudo apresenta um maior enfoque na análise do comportamento de aquecedores de gás indireto a banho d'água e na regulagem dinâmica da temperatura de entrega através de um controlador PID que comanda a válvula de três vias. Investigou-se o desempenho de uma estação de entrega de gás natural existente baseada nas condições operacionais estabelecidas no projeto. Simulou-se o escoamento correspondente a operação em uma estação de entrega real e realizou-se comparação direta com dados de instrumentação enviados ao supervisório de controle e aquisição de dados (SCADA). A metodologia desenvolvida reproduziu de forma adequada a operação em condições normais da estação de entrega de São Carlos-SP e eventos anormais como cenários de apagamento dos aquecedores que foram registrados no banco de dados do supervisório. Finalmente analisaram-se procedimentos a serem aplicados nas estações de entrega existentes para redução do consumo do gás combustível utilizado no processo de pré-aquecimento.

\section{Palavras-chave}

Estações de Entrega de Gás Natural; Simulação de Processos Dinâmicos; Aquecedores de Gás Indiretos de Banho de Água. 


\section{Abstract}

Barreto, Claudio Veloso; Nieckele, Angela O. Nieckele (Advisor); Pires, Luis Fernando G. (Co-Advisor) Numerical Simulation of Natural Gas Flow in Citygate Stations, 2011. 139p. MSc. Dissertation - Departamento de Engenharia Mecânica, Pontifícia Universidade Católica do Rio de Janeiro.

Numerical simulation of the dynamic process of natural gas flow through citygate stations, considering the transient behavior of the main existing equipments, such as three-way-valves, pressure reduction valves and gas heaters. Development of the dynamic control logic (PID controller) of all relevant equipments. To determine the natural gas thermodynamic properties, the equations of state of Peng-Robinson and Soave-Redlich-Kwong were employed. The study concentrates in the analysis of indirect fired water bath heater and in the delivery temperature regulation using a PID controller that commands the three-way-valve. It was investigated the performance of an existing natural gas citygate station based on established design operational conditions. The flow field corresponding to an operational natural gas city gate station was determined and direct comparison with instrumentation data sent to supervisory control and data acquistion (SCADA) was performed. The methodology developed reproduced in a satisfactory way the normal operational condition of the São Carlos-SP natural gas citygate station and abnormal events such as the heater shut-down scenarios registered on SCADA database. Finally, procedures to be applied in existing delivery stations for the reduction of gas fuel consumption in the pre-heating process were analyzed.

\section{Keywords}

Natural Gas Citygate Stations; Dynamic Process Simulation; Indirect Fired Water Bath Heaters. 


\section{Sumário}

$\begin{array}{ll}\text { 1. Introdução } & 21\end{array}$

1.1. Estação de Entrega de Gás Natural $\quad 24$

$\begin{array}{lll}\text { 1.1.1. } & \text { Filtragem } & 24\end{array}$

$\begin{array}{lll}\text { 1.1.2. } & \text { Aquecimento } & 26\end{array}$

1.1.3. Redução e Controle de Pressão 26

1.1.4. Controle de Gás Combustível 27

1.1.5. Medição 28

1.2. Objetivo 28

1.3. Metodologia 29

1.4. Organização do Manuscrito 30

2. Revisão Bibliográfica 31

3. Propriedades Termodinâmicas 34

3.1. Equações de Estado 34

3.1.1. Equação de Soave-Redlich-Kwong 35

3.1.2. Equação de Peng-Robinson 36

3.1.3. Propriedades de Mistura 37

3.1.3.1. Regra de Combinação Clássica de Van de Waals 37

3.1.3.2. Propriedades de Substância Pura e de Mistura 38

3.2. Viscosidade Absoluta $\quad 39$

3.3. Propriedades Térmicas $\quad 39$

3.3.1. Processo Termodinâmico com Variação de Pressão e Temperatura 41

3.3.2. $\quad$ Processo Isobárico à Pressão Zero (Gás Perfeito) 42

3.3.3. Processo Isotérmico 46

3.4. Processo Iso-entálpico e Efeito Joule-Thompson 46 
4. Modelagem Matemática e Numérica 48

4.1. Balanço nos Nós 49

4.2. Modelos de Equipamentos Estáticos, Dinâmicos e Transientes 50

4.3. Condições de Contorno: Pontos de Entrada e Saída de Gás 51

4.4. Válvulas de Controle para Redução de Pressão 52

4.4.1. Curvas Características de Válvulas de Controle $\quad 54$

4.5. Filtros Ciclones $\quad 56$

4.6. Dutos Cilíndricos Aéreos $\quad 57$

4.7. Aquecedores/Resfriadores Simplificado $\quad 61$

4.8. Aquecedores Indiretos de Banho 62

4.8.1. Temperatura de saída do aquecedor (TSAIB) 65

4.8.2. Temperatura da água usando Runge-Kutta (TARK4) 66

4.8.3. Controle de Duas Posições de Acendimento da Chama 67

4.9. Válvulas de Três Vias de Fluxo Divergente 68

4.10. Controlador Proporcional-Derivativo-Integrativo 69

4.11. Método para Solução do Sistema de Equações Não Lineares 71

5. Validação do Simulador de Estações de Entrega 74

5.1. Efeito Joule-Thomson nas Válvulas e Fluxo de Calor Requerido nos $\begin{array}{ll}\text { Aquecedores. } & 74\end{array}$

5.2. Cálculo da vazão e da queda de pressão num escoamento adiabático compressível $\quad 82$

6. Modelagem de uma Estação de Entrega de Gás Natural Existente 85

6.1. Condição de Entrada e Composição do Gás Natural 85

6.2. Descrição dos Equipamentos $\quad 88$

$\begin{array}{ll}\text { 6.2.1. Filtro Ciclone } & 88\end{array}$

6.2.2. Válvula de Três Vias $\quad 89$

6.2.3. Aquecedores Indiretos de Gás de Banho de Água 90 
6.2.4. Tubulação de 6" na Saída do By-pass dos Aquecedores

6.2.5. Tubulação de 8" entre Aquecimento e a Redução de Pressão 93

6.2.6. Válvula Redutora de Pressão - Monitora 94

6.2.7. Válvula Redutora de Pressão - Reguladora 95

6.3. Comparação com Condições Operacionais de Projeto 97

6.4. Cenários Dinâmicos na Estação de Entrega sem

Malha de Controle de Temperatura (comparação com dados do SCADA) 99

6.4.1. Operação com Dois Aquecedores 100

6.4.2. Apagamento de um Aquecedor 105

7. Cenários Dinâmicos com Malha de Controle de Temperatura 110

7.1. Malha de Controle Existente 110

7.1.1. Controlador Tipo PI para Temperatura de Entrega (TIC31) 111

7.1.2. Cenário com Dois Aquecedores - Vazão Constante 112

7.1.3. Controlador de Duas Posições para Temperatura

do Banho (TIC60A/B) 113

7.1.4. Cenário com Dois Aquecedores - Variação da Vazão de Entrega 114

7.2. Malha de Controle de Temperatura Modificada 116

7.2.1. Controlador PI de Vazão de Combustível (TIC33) 117

7.2.2. Cenário com Dois Aquecedores - Vazão Constante 117

7.2.3. Cenário com Dois Aquecedores - Variação da Vazão de Entrega 119

8. Conclusões e Recomendações 121

$\begin{array}{ll}\text { Referências Bibliográficas } & 124\end{array}$

$\begin{array}{ll}\text { Apêndice A. Descrição do Simulador } & 127\end{array}$

$\begin{array}{ll}\text { A.1. Rotinas Iterativas e Módulos de Solução } & 127\end{array}$

A.2. Demonstração da Rotina Iterativa Estática 131

A.2.1. Módulo de Pressão - Caso de Teste 132

A.2.2. Módulo de Temperatura - Caso de Teste 133 
A.2.3. Módulo de Composição - Caso de Teste

A.2.4. Resultados Obtidos no Caso de Teste

Apêndice B. Catálogos e Folha de Dados dos Equipamentos 


\section{Lista de figuras}

Figura 1.1 - Malha de Gasodutos do Brasil (ANP, 2010)

Figura 1.2 - Foto da Estação de Entrega de São Carlos

da TBG $(22 / 05 / 2010)$

Figura 1.3 - Esquemático de uma Estação de Entrega de Gás 25

Figura 1.4 - Esquemático da Área de Redução de Pressão

Figura 3.1 - Processo termodinâmico com qualquer variação de pressão e temperatura

Figura 3.2 - Algorítmo de cálculo da temperatura variando pressão e entalpia $\mathrm{Tq}(\mathrm{p}, \mathrm{h})$

Figura 3.3 - Variação do calor molar com a temperatura (Gás perfeito)

Figura 3.4 - Variação da entalpia molar com a temperatura (Gás perfeito)

Figura 3.5 - Variação da entropia molar com a temperatura (Gás Perfeito)

Figura 3.6 - Algorítmo de cálculo da temperatura no processo

isobárico a pressão zero $\mathrm{Tp}(\mathrm{h})$

Figura 3.7 - Comparação de curvas de inversão Joule-Thompson do metano

Figura 4.1 - Balanço em um nó com cinco elementos

Figura 4.2 - Condições de Contorno: Pontos de Entrada e Saída de Gás

Figura 4.3 - Modelo Dinâmico da Válvula de Controle

Figura 4.4 - Tipos de Obturadores de Válvula de Controle

Globo [Fonte: Valvugás]

Figura 4.5 - Curvas Características de Válvula de Controle

Figura 4.6 - Filtro ciclone em corte com indicação das dimensões

Figura 4.7 - Modelo Estático do Filtro Ciclone

Figura 4.8 - Modelo do Duto Aéreo

Figura 4.9 - Coeficiente Externo de Troca para Dutos Aéreos

$($ Dex $=8 "$ e Prar $=0,71)$ 
Figura 4.10 - Modelo do Aquecedor/Resfriador Simplificado

Figura 4.11 - Visão em Corte do Aquecedor Indireto de Banho 63

Figura 4.12 - Modelo Esquemático Aquecedor Indireto de Banho 63

Figura 4.13 - Eficiência de Combustão em Aquecedores de Chama Indireta

(Fonte: API-12K, Apêndice C, pág 22)

Figura 4.14 - Algoritmo da Temperatura de Saída do Aquecedor Indireto (TSAIB)

Figura 4.15 - Algoritmo da Temperatura da Água usando Método RK-4 (TARK4)

Figura 4.16 - Lógica de Controle de Duas Posições de

Acendimento da Chama

Figura 4.17 - Modelo esquemático da válvula de três vias (esquerdo)

e visão em corte (direiro) [Fonte: Válvugás]

Figura 4.18 - Curvas de Igual Percentagem para Cv1 e Cv2

(Válvula de Três Vias Divergente)

Figura 4.19 - Diagrama de um Controlador PID Convencional

Figura 4.20 - Controlador de Temperatura de Entrega

(Sistema de Pré-Aquecimento)

Figura 4.21 - Algoritmo do Método de Newton - Pacote KINSOL 73

Figura 5.1 - Diagrama esquemático do exemplo 1

Figura 5.2 - Ganho de Temperatura vs Fluxo de Calor

no Aquecedor (Peng-Robinson)

Figura 5.3 - Ganho de Temperatura vs Fluxo de Calor no Aquecedor (Soave-Redlich-Kwong)

Figura 5.4 - Queda de Temperatura vs Queda de Pressão na Válvula (Peng-Robinson)

Figura 5.5 - Queda de Temperatura vs Queda de Pressão

na Válvula (Soave-Redlich-Kwong)

Figura 5.6 - Curvas isotermas para o fator de compressibilidade

(Gás 1 - Peng-Robinson)

Figura 5.7 - Curvas isotermas para o fator de compressibilidade

(Gás 3 - Peng-Robinson) 
Figura 5.8 - Coeficiente J-T vs Pressão de Entrada (Gás 1) 80

Figura 5.9 - Coeficiente J-T vs Pressão de Entrada (Gás 3) 80

Figura 5.10 - Coeficiente J-T vs Temperatura de Entrada (Gás 1) 81

Figura 5.11 - Coeficiente J-T vs Temperatura de Entrada (Gás 3) 81

Figura 5.12 - Diagrama esquemático do exemplo 2 (Parâmetro de Entrada) 83

Figura 6.1- Esquemático do Modelo de Simulação da Estação

de Entrega de São Carlos

Figura 6.2- Filtro Ciclone FT01 88

Figura 6.3- Válvulas Controle de Três Vias TV31A/B 89

Figura 6.4 - Curva CV X Fração de Abertura para Saída 1 - Válvula

PARCOL 6pol 90

Figura 6.5 - Curva CV X Fração de Abertura para Saída 2 - Válvula

PARCOL 6pol 90

Figura 6.6 - Aquecedores de Gás F01A/B 91

Figura 6.7 - Curva CV X Fração de Abertura- Válvula Reflux 819 (3”) 95

Figura 6.8 - Curva CV X Fração de Abertura- Válvula Aperflux 851 (3”) 96

Figura 6.9.- Resultados do Regime Permanente na Condição Máxima 98

Figura 6.10 - Cenário de Temperatura de Entrada e Vazão de Saída da EE 101

Figura 6.11 - Cenário de Vazão de Combustível nos Aquecedores F01A/B 101

Figura 6.12 - Históricos de temperatura na saída do by-pass

dos aquecedores (SIMGAS e SCADA - 02 aquecedores) 102

Figura 6.13 - Históricos de temperatura da água nos aquecedores

(SIMGAS e instrumentos locais - 02 aquecedores) 102

Figura 6.14 - Históricos de temperatura de entrada e saída do HEAD8

(SIMGAS e SCADA)

Figura 6.15 - Históricos de temperatura de entrega (SIMGAS e SCADA) 104

Figura 6.16 - Variação do coeficiente JT com a temperatura de entrega

(SIMGAS e SCADA - 02 aquecedores)

Figura 6.17 - Cenário de Temperatura de Entrada (Linha Tronco) 
Figura 6.19 - Históricos de temperatura na saída do by-pass dos aquecedores (Apagamento do aquecedor F01A)

Figura 6.20 - Históricos de temperatura da água dos aquecedores (Apagamento do aquecedor F01A)

Figura 6.21 - Históricos de temperaturas na entrada e na saída do HEAD8 (Apagamento do aquecedor F01A)

Figura 6.22 - Históricos de temperaturas de entrega

(Apagamento do aquecedor F01A)

Figura 7.1 - Diagrama da Malha de Controle de Temperatura Existente

Figura 7.2 - Histórico de vazão e temperaturas nos aquecedores e na entrada da PCV12A (Malha de Controle Existente - Dois Aquecedores)

Figura 7.3 - Histórico de temperatura de entrega e fração de abertura das válvulas TV31 A/B (Malha de Controle Existente - Dois Aquecedores)

Figura 7.4 - Histórico de consumo de combustível nos aquecedores

(Malha de Controle Existente - Dois Aquecedores)

Figura 7.5 - Histórico de vazão e temperaturas nos aquecedores e na entrada da PCV12A (Malha Existente - Variação da Vazão de Entrega)

Figura 7.6 - Histórico de temperatura de entrega e fração de abertura das válvulas TV31A/B (Malha Existente - Variação da Vazão de Entrega)

Figura 7.7 - Histórico de consumo de combustível nos aquecedores (Malha Existente - Variação da Vazão de Entrega)

Figura 7.8 - Diagrama da Malha de Controle de Temperatura Modificada

Figura 7.9 - Histórico de vazão e temperaturas nos aquecedores e na entrada da PCV12A (Malha de Controle Modificada - Dois Aquecedores)

Figura 7.10 - Histórico de temperatura de entrega e fração de abertura das válvulas TV31 A/B (Malha de Controle Modificada - Dois Aquecedores)

Figura 7.11 - Histórico de consumo de combustível nos aquecedores (Malha de Controle Modificada - Dois Aquecedores)

Figura 7.12 - Histórico de vazão e temperaturas nos aquecedores e na entrada da PCV12A (Malha Modificada - Variação da Vazão de Entrega) 
Figura 7.13 - Histórico de temperatura de entrega e fração de abertura das válvulas TV31A/B (Malha Modificada - Variação da Vazão de Entrega)

Figura 7.14 - Histórico de consumo de combustível nos aquecedores (Malha Modificada - Variação da Vazão de Entrega)

Figura A.1 - Fluxograma de Ações - Rotina Interativa Estática

Figura A.2 - Fluxograma de Ações - Rotina Iterativa Dinâmica

Figura A.3 - Parâmetros de Entrada do Caso de Teste

Figura B.1 - Catálogo da Válvula de Controle Pietro Fiorentini (www.fiorintini.com).

Figura B.2- Catálogo da Válvula de Três Vias PARCOL 1-8113 (www.parcol.com)

Figura B.3 - Folha de Dados dos Aquecedores F01A/B 


\section{Lista de tabelas}

Tabela 1.1 - Estações de Entrega de Gás Natural do GASBOL 23

Tabela 1.2 - Tipos de Estação de Entrega de Gás por Capacidade 24

Tabela 3.1 - Propriedades de Substância Pura das Espécies Químicas 38

Tabela 4.1 - Classificação e Variáveis de Cenários dos

Modelos de Equipamentos 51

Tabela 4.2 - Tipos de filtros ciclones e suas relações geométricas 56

Tabela 4.3 - Parâmetros empíricos para dimensionamento de gasodutos $\quad 59$

Tabela 5.1 - Frações Molares das Composições de Gás Natural

do Exemplo 1

Tabela 5.2 - Comparação entre simuladores SIMGAS

e TGNET (exemplo 2) $\quad 84$

Tabela 6.1 - Composição do Gás Natural do GASBOL (20-05-2010) 86

Tabela 6.2 - Propriedades do Gás Calculadas pelo SIMGAS

e obtidas do SCADA $\quad 88$

Tabela 6.3 - Regime Permanente para Condição Máxima Operacional 99

Tabela 7.1 - Cenário de Variação de Vazão de Entrega 114

Tabela A.1 - Critério de Convergência dos Módulos de Solução 130

Tabela A.2 - Erro Máximo por Iteração (Caso de Teste) 135

Tabela A.3 - Resultados nos Aquecedores (Caso de Teste) 136

Tabela A.4 - Resultados nos Pontos de Entrada e Saída (Caso de Teste) 136 


\section{Nomenclatura}

$\rho$ Massa específica $\left(\mathrm{kg} / \mathrm{m}^{3}\right)$

$p$ Pressão absoluta $(\mathrm{Pa})$

$T$ Temperatura absoluta (K)

$M_{g}$ Massa molecular do gás de mistura $(\mathrm{kg} / \mathrm{kmol})$

$Z$ Fator de compressibilidade

$\Re$ Constante universal dos gases perfeitos. $\mathfrak{R}=8,3145 \mathrm{~J} / \mathrm{mol} . \mathrm{K}$

$v$ Volume específico $\left(\mathrm{m}^{3} / \mathrm{kg}\right)$

$v$ Volume específico molar $\left(\mathrm{m}^{3} / \mathrm{kmol}\right)$

$a, b, m$ Coeficientes da equação de estado

$p_{c}$ Pressão crítica absoluta $(\mathrm{Pa})$

$T_{c}$ Temperatura crítica absoluta (K)

$\omega$ Fator acêntrico

$A$, $B$ Coeficientes da equação de estado na forma $\mathrm{Z}(\mathrm{p}, \mathrm{T})$

$\phi_{g} \quad$ Propriedade de mistura do gás

$\phi_{i} \quad$ Propriedade da espécie $i$

$X$ Fração ou percentual molar

$k_{i j}$ Constante empírica de iteração binária

$a_{g}$ Coeficiente $a$ da equação de estado para mistura

$a_{i}, a_{j} \quad$ Coeficiente $a$ da equação de estado espécie $i$, espécie $j$

$X_{i}, X_{j}$ Fração molar para espécie $i$, espécie $j$

$\mu_{g} \quad$ Viscosidade absoluta do gás $(\mathrm{cP})$

$A_{\mu}, B_{\mu}, C_{\mu} \quad$ Coeficientes da viscosidade

$u$ Energia interna molar $(\mathrm{kJ} / \mathrm{kmol})$ ou específica $(\mathrm{kJ} / \mathrm{kg})$

$h$ Entalpia molar $(\mathrm{kJ} / \mathrm{kmol})$ ou específica $(\mathrm{kJ} / \mathrm{kg})$

$s$ Entropia molar $(\mathrm{kJ} / \mathrm{kmol} . \mathrm{K})$ ou específica $(\mathrm{kJ} / \mathrm{kg} . \mathrm{K})$

$T_{\text {ref, }} \quad$ Temperatura de referência $\left(\mathrm{T}_{\text {ref, }}=300 \mathrm{~K}\right)$

$p_{\text {ref, }} \quad$ Pressão de referência $\left(\mathrm{p}_{\text {ref, }}=101325 \mathrm{~Pa}\right)$

$T_{\text {std }}$ Temperatura padrão $\left(\mathrm{T}_{\mathrm{std}}=20 \mathrm{oC}\right)$

$\Delta u$ Variação de energia interna molar $(\mathrm{kJ} / \mathrm{kmol})$

$\Delta h$ Variação de entalpia molar $(\mathrm{kJ} / \mathrm{kmol})$ 
$\Delta s \quad$ Variação de entropia molar $(\mathrm{kJ} / \mathrm{kmol} . \mathrm{K})$

iter Contador de iteração numérica

$C_{p}$ Calor molar a pressão constante (kJ/kmol.K) ou específico (kJ/kg.K)

$C_{1} \ldots C_{5}$ Coeficientes do calor molar

$\Delta h_{p}$ Variação de entalpia molar - processo a pressão zero $(\mathrm{kJ} / \mathrm{kmol})$

$\Delta u_{p}$ Variação de energia interna - processo a pressão zero $(\mathrm{kJ} / \mathrm{kmol})$

$\Delta h_{T}$ Variação de entalpia molar - processo isotérmico $(\mathrm{kJ} / \mathrm{kmol})$

$\Delta u_{T}$ Variação de energia interna - processo isotérmico $(\mathrm{kJ} / \mathrm{kmol})$

$C_{v} \quad$ Calor molar a volume constante $(\mathrm{kJ} / \mathrm{kmol} . \mathrm{K})$ ou específico $(\mathrm{kJ} / \mathrm{kg} . \mathrm{K})$

$\mu_{J T}$ Coeficiente Joule-Thomson

$p_{s t d,} \quad$ Pressão padrão $\left(\mathrm{p}_{\mathrm{std}}=1 \mathrm{~atm}=101325 \mathrm{~Pa}\right)$

$\dot{M} \quad$ Vazão molar (kmol/s)

$M_{i} \quad$ Massa molecular da espécie i $(\mathrm{kg} / \mathrm{kmol})$

$M_{a r} \quad$ Massa molecular do ar seco $\left(\mathrm{M}_{\mathrm{ar}}=28,9626 \mathrm{~kg} / \mathrm{kmol}\right)$

$\dot{m} \quad$ Vazão em massa $(\mathrm{kg} / \mathrm{s})$

$\rho_{\text {std }}$ Massa específica padrão $\left(\mathrm{kg} / \mathrm{Sm}^{3}\right)$

$Q_{s t d}, Q$ Vazão volumétrica padrão $\left(\mathrm{Sm}^{3} / \mathrm{s}\right) .\left(1 \mathrm{Sm}^{3} / \mathrm{s}=86.4 \mathrm{SMm}^{3} / \mathrm{dia}\right)$

$q_{g} \quad$ Fluxo de calor transferido ao gás $(\mathrm{kJ} / \mathrm{s})$

$f_{o} \quad$ Fração de abertura

$T_{\infty} \quad$ Temperatura ambiente $\left({ }^{\circ} \mathrm{C}\right)$

$T_{a}$ Temperatura da água $\left({ }^{\circ} \mathrm{C}\right)$

$T a_{\text {máx }} \quad$ Temperatura máxima da água $\left({ }^{\circ} \mathrm{C}\right)$

$T a_{m i n} \quad$ Temperatura mínima da água $\left({ }^{\circ} \mathrm{C}\right)$

Kp Coeficiente de perda de pressão

$C v$ Coeficiente de vazão da válvula $\left(\mathrm{gpm} / \mathrm{psi}^{0.5}\right)$

$\gamma_{g}$ Densidade do gás relativa ao ar $\left(\mathrm{M}_{\mathrm{g}} / \mathrm{M}_{\mathrm{ar}}\right)$.

$\lambda_{g}$ Razão de calores específicos do gás $\left(\mathrm{C}_{\mathrm{p}} / \mathrm{C}_{\mathrm{v}}\right)$.

$Y$ Fator de expansão do gás

$\lambda_{a r}$ Razão de calores específicos do ar à $60 \mathrm{oF}$ e $1 \mathrm{~atm} . \lambda_{\mathrm{ar}}=1,4$

$F_{\lambda}$ Razão de calores específicos do gás e do ar

$D$ Diâmetro externo (m)

$D_{i} \quad$ Diâmetro interno (m)

$\Delta p \quad$ Diferencial de pressão no elemento $(\mathrm{kPa})$ 
$g$ Aceleração da gravidade. $\mathrm{g}=9,80665 \mathrm{~m} / \mathrm{s}^{2}$.

$v_{1}$ Velocidade na entrada do elemento $(\mathrm{m} / \mathrm{s})$

$v_{2}$ Velocidade na saída do elemento $(\mathrm{m} / \mathrm{s})$

$q_{\infty}$ Fluxo de calor transferido ao ambiente $(\mathrm{kJ} / \mathrm{s})$

$L$ Comprimento do duto (m)

$R u \quad$ Rugosidade superficial (m)

$f$ Fator de atrito de Colebrook

U Coeficiente global de transferência de calor $\left(\mathrm{W} / \mathrm{m}^{2} . \mathrm{K}\right)$

$\mathrm{Rt}_{\text {aço }}$ Resistência térmica do duto de aço $\left(\mathrm{m}^{2} . \mathrm{K} / \mathrm{W}\right)$

$\mathrm{Rt}_{\text {rev }}$ Resistência térmica dos revestimentos $\left(\mathrm{m}^{2} . \mathrm{K} / \mathrm{W}\right)$

$\mathbf{k}_{\text {aço }}$ Condutividade térmica do aço (W/m.K)

$\mathbf{h}_{\mathbf{i}}$ Coeficiente interno de troca de calor por convecção $\left(\mathrm{W} / \mathrm{m}^{2} . \mathrm{K}\right)$

$\mathbf{h}_{\text {ex }}$ Coeficiente externo de troca de calor por convecção $\left(\mathrm{W} / \mathrm{m}^{2} . \mathrm{K}\right)$

Pr Número de Pradtl

$\mathbf{N u}_{\mathbf{i}} \quad$ Número de Nusselt Interno

$\mathbf{N u}_{\mathrm{ex}} \quad$ Número de Nusselt Externo

Re Número de Reynolds

qa Calor transferido da água para o gás $(\mathrm{kJ} / \mathrm{s})$

$q b$ Calor de combustão $(\mathrm{kJ} / \mathrm{s})$

$m_{a} \quad$ Massa de água $(\mathrm{kg})$

$C p_{a} \quad$ Calor específico da água $(\mathrm{kJ} / \mathrm{kg})$

$\mathrm{PCI}_{g}$ Poder calorífico inferior molar do gás

$\eta_{b}$ Eficiência de combustão

$\dot{m}_{b} \quad$ Fluxo de massa do gás de combustão $(\mathrm{kg} / \mathrm{s})$

$E(t) \quad$ Erro do controlador

$S(t) \quad$ Setpoint do controlador

$C(t)$ Variável de controle

$X(t)$ Resposta do controlador

$K_{C}$ Constante de proporcionalidade do controlador

$K_{I}$ Constante de integração do controlador

$K_{D}$ Constante derivativa do controlador

Bo Bias do controlador

$P(t) \quad$ Resposta proporcional do controlador 
I(t) Resposta integral do controlador PID

$D(t)$ Resposta derivativa do controlador PID

\title{
Subscritos
}

\author{
c Ponto Crítico \\ $g$ Propriedade da mistura de gás \\ std Padrão ou standard \\ $i, j \quad$ Índice da espécie química \\ $e$ Entrada do elemento \\ $s \quad$ Saída do elemento \\ ar Ar seco \\ máx Máximo \\ mín Mínimo \\ JT Joule-Thomson \\ $\mu$ Viscosidade \\ ref Referência \\ $\infty$ Ambiente \\ set Ponto de ajuste ou setpoint \\ in Interno \\ ex Externo
}

